\title{
Sliding mode plus U-control method for continuous time SISO dynamic systems
}

\section{Zhengrong Wei*, Ruobing Li and Quanmin Zhu}

Department of Engineering Design and Mathematics, University of the West of England,

Bristol, England, UK

Email: Zhengrong2.Wei@live.uwe.ac.uk

Email: Ruobing2.Li@live.uwe.ac.uk

Email: quan.zhu@uwe.ac.uk

*Corresponding author

\begin{abstract}
This paper proposes a new sliding mode augmented U-model-based control method ( $\mathrm{U}_{\mathrm{SM}^{-}}$-control) for controlling a class of Single-Input SingleOutput (SISO) dynamic systems with internal uncertain parameters and external system noise/disturbance. To implement this idea, this study firstly introduces the U-model definition, establishes the corresponding $\mathrm{U}_{\mathrm{SM}^{-}}$-controlbased system design framework, and explains its design procedure step by step. Then this study selects a simplified nonlinear helicopter model for simulation case studies, using Matlab/Simulink to test and demonstrate the proposed $\mathrm{U}_{\mathrm{SM}^{-}}$-control method in terms of tracking ability, and finally discusses the comparison results generated from $\mathrm{U}_{\mathrm{SM}^{-}}$-control and $\mathrm{U}$-control approaches to test the stability and robustness of proposed control method in this study.
\end{abstract}

Keywords: U-model; U-model-based control; sliding mode control; $\mathrm{U}_{\mathrm{SM}^{-}}$-control; simulation demonstrations.

Reference to this paper should be made as follows: Wei, Z., Li, R. and Zhu, Q. (2021) 'Sliding mode plus U-control method for continuous time SISO dynamic systems', Int. J. Cybernetics and Cyber-Physical Systems, Vol. 1, No. 1, pp.68-92.

Biographical notes: Zhengrong Wei received his bachelor's degree from Shanghai Jiaotong University. Now he is a postgraduate in University of the West of England. He majors in Master in Research Engineering now.

Ruobing Li received his bachelor's degree from Northwestern Polytechnical University, and received his MSc from University of Bristol. Now he is a $\mathrm{PhD}$ in University of the West of England.

Quanmin Zhu received his MSc from Harbin Institute of Technology in China in 1983, and his PhD from University of Warwick in UK in 1989. Now he is a Professor in Control Systems, with extensive research experiences in the field of nonlinear system modelling, identification, and control, and electroacupuncture instrumentation in University of the West of England. He has published over 170 papers on these topics. In the meantime, he is the editor/founder of International Journal of Modelling, Identification and Control, and the editor of International Journal of Computer Applications in Technology. 


\section{Introduction}

Plant modelling has played a very critical role in model-based control system design. In general, there are two main important considerations for model utility and control systems, 1) approximation capabilities in terms of accuracy and conciseness; 2) control oriented structure for control system formation and calculation.

Regarding the first concern, in practice, even for academic research, seldom does a model have accurate representation to real plants, therefore model-based control system design should take internal uncertainty and external disturbance like system error into consideration (Oberkampf et al., 2004). Sliding Mode Control (SMC) (Edwards and Spurgeon, 1998) has been effectively used to deal with uncertainties in way of variable structure system, that is, a control system involving discontinuous control actions. Sliding mode control has developed in various sliding manifolds such as integral sliding mode control approach (Wang et al., 2017), second-order sliding mode control approach (Ding et al., 2017), super-twist sliding mode control approach (Chalanga et al., 2016), and adaptive sliding mode control approach (Li et al., 2016). SMC method is a special kind of non-linear control with the characteristics of control discontinuity, even though the switching characteristics of the control system structure change with time. In summary, the advantages of SMC method are that firstly the dynamic behaviour of the system may be tailored by the particular choice of the switching function (Edwards and Spurgeon, 1998) and secondly even if the system has external disturbances and uncertain parameters, the sliding mode control method can still keep system stability and have strong robustness.

For the second concern, the key idea used in this study is U-model-based control method, U-control method in short. U-model represents a class of smooth systems which use time-varying parameters and functions to absorb all other items except system input $u$ and its relative high order derivative terms. Generally, this unnoticed and new U-model can be converted and expressed into single-layer and multi-layer, that is, polynomial model and state space model from linear and non-linear systems. Zhu et al. (1991) firstly proposed the basic designing procedure for controller in U-model, that is, obtaining controller output $u$ from solving the root of the controller output function by using Newton-Raphson algorithm. Zhu and Guo (2020) generally indicated the polynomial definition of U-model and introduced U-control system framework and design procedure by pole placement method, which also provides general converting procedure from normal smooth linear/nonlinear systems into U-model-based expression. Then, U-model expands the Non-linear Autoregressive Moving Average model with Exogenous inputs (NARMAX) function as power series in the current control term, thus allowing simple polynomial root solving procedures to be used for controller synthesis. Generally, U-control approach can save the computation of control system design procedure from avoiding nonlinear system modelling linearisation processing. However, U-model-based dynamic inversion is very sensitive to internal uncertainties as well as the controlled performance. In this case, reducing U-control method's sensitivity to uncertainty, that is, improving its robustness is a hot research issue. 
In the past 20 years, Discrete Time (DT) U-model and U-control systems have been well studied due to the easy implementation of model inversion. Representatively, these approaches include U-model-based pole placement control system design method (Zhu, 1989; Zhu et al., 2015), U-Smith predictor design for system with input time delay (Geng et al., 2019), adaptive U-model-based control method for controlling total nonlinear dynamic systems (Zhu et al., 2018), U-model-based neural network enhanced control method (Zhu et al,, 2019a), adaptive U-model-based control method for controlling underactuated coupled nonlinear multivariable unmanned marine robotics (Hussain et al., 2020). However, the majority of U-control approaches have assumed an accurate plant model and perfectly matched system available. Therefore, U-model-based robustness control method is quite necessary and challenging in development. Meanwhile, Continuous Time (CT) U-control system design is less attended (Zhu et al., 2019b). This is because it is difficult in the solution of CT dynamic inversion with high order derivative terms of controlled system input and output. Consequently, this study takes these two challenges to try a solution for CT U-model-based robust control system design, that is, in the form of combined U-control method's computation saving capacity and SMC method's strong robustness.

Accordingly, the main contribution of this study is justified with

1) Proposal of a new $U_{S^{-}}$-control framework integrating U-control and SMC method, which accommodates both control-oriented model structure and strong robustness against imperfect model representation.

2) Computational experiments by Simulink to bench test the developed control system design procedure. In addition, the exemplary case study provides potential readers/users a routine for their ad hoc research expansion and applications.

The rest of the study is organised as follows: Section 2 firstly introduces the U-model realisation of general classical polynomial and state space models, then shows U-control system design framework under perfectly matched and mismatched situations. Section 3 presents $\mathrm{U}_{\mathrm{SM}}$-control system design framework and its relative control system design procedures. Based on Section 3, Section 4 introduces the compensated plant inverter and dynamic inverter design procedure in $\mathrm{U}_{\mathrm{SM}}$-control system step by step. Then, based on the theory provided in Sections 3 and 4, Section 5 selects a case study of pitch angle control with a helicopter, provides computational experiments to demonstrate the theoretical results numerically, and compares the simulated results of from U-control method and $\mathrm{U}_{\mathrm{SM}}$-control method. Section 6 draws the conclusion and future work of this study.

\section{U-model and U-control}

\subsection{U-polynomial (Zhu et al., 2019b)}

For a SISO U-polynomial model, $U_{p}$ in short with a triplet of $(y, u, \lambda) \in U_{p}$,

$$
U_{p}: \begin{gathered}
(M) \\
y
\end{gathered}=\sum_{j=0}^{J} \lambda_{j}\left(\begin{array}{c}
(N) \\
u
\end{array}\right)^{j}, M \geq N
$$


where $\begin{gathered}(M) \\ y\end{gathered}$ is the $M$-th order derivatives of the plant output $y$ and $\begin{gathered}(N) \\ u\end{gathered}$ is the $N$-th order derivatives of the controller output $u . \lambda_{j}\left(Y_{M-1}, U_{N-1},\right) \in \mathbb{R}^{+}$is the time-varying function absorbing all the other terms except the input $\left(\begin{array}{c}(N) \\ u\end{array}\right)^{j}$ in

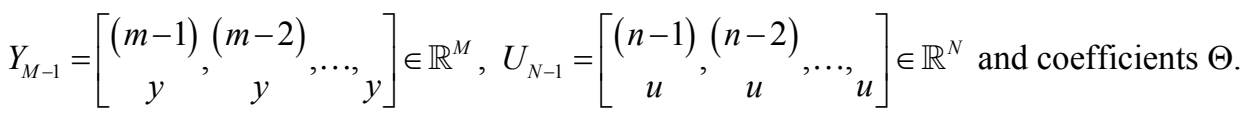

Remark 1: U-polynomial is an alternative expression of classical polynomials, with timevarying parameters and control-oriented structure.

Consider a classical NARMAX polynomial model as an example to show its U-realisation procedure:

$$
\ddot{y}=-\dot{y}+(1+y) u+u^{2}+y+3 u^{3}
$$

where its relative U-model-based expression is:

$$
\left\{\begin{array}{l}
\ddot{y}=\lambda_{0}+\lambda_{1} u+\lambda_{2} u^{2}+\lambda_{3} u^{3} \\
\lambda_{0}=-\dot{y}+y \\
\lambda_{1}=1+y \\
\lambda_{2}=1 \\
\lambda_{3}=3
\end{array}\right.
$$

Inspection of (2) and (3) shows that the U-polynomial is a direct realisation of classical polynomials. It should be explained that the U-polynomial is the same as its classical polynomials in the model properties, but a general concise structure for control system design, particularly non-linear systems (Zhu et al., 2015).

Remark 2: For converting classical polynomial into U-polynomial, an absorbing rule (Zhu et al., 2019b) is generally applied, that is, to absorb all associated with each input $u$ function into a time variant coefficient $\lambda_{j}$.

Remark 3: It is clear for degree $J=1$ in U-polynomial model (1), it is simplified into a linear in the input $U$ polynomial expression

$$
\begin{gathered}
(M) \\
y
\end{gathered}=\lambda_{0}+\lambda_{1}\left(\begin{array}{c}
(N) \\
u
\end{array}\right), M \geq N
$$

\subsection{U-Rational model-expanded U-polynomial (Li et al., 2020)}

U-Rational model, $U_{R}$ in short, can be defined as the ratio of two U-polynomial models, which can be expressed as: 


$$
U_{R}: \begin{aligned}
& (M) \\
& y
\end{aligned}=\frac{U_{p n}}{U_{p d}}=\frac{\sum_{j=0}^{n} \lambda_{n, j} f_{n, j}\left(\begin{array}{c}
(N) \\
u
\end{array}\right)}{\sum_{j=0}^{d} \lambda_{d, j} f_{d, j}\left(\begin{array}{c}
(N) \\
u
\end{array}\right)}, M \geq N
$$

where $f_{n j}$ is a smooth function absorbing the controller output vector $\begin{gathered}(N) \\ u\end{gathered}$ in numerator and $f_{d j}$ can absorb it in denominator. $n j$ and $d j$ can absorb all the other terms except controller output vector $(N)$ in numerator and denominator respectively. Consider a simple example to show the converting procedure from a classical rational model to U-model:

$$
\dot{y}=\frac{1+y^{2}+u+0.5 u^{3}}{e^{-y}+\cos (y)+y+u^{2}}
$$

Accordingly, its expanded U-polynomial is

$$
\dot{y}=\frac{\lambda_{n 0}+\lambda_{n 1} f_{n 1}(u)+\lambda_{n 2} f_{n 2}(u)}{\lambda_{d 0}+\lambda_{d 1} f_{d 1}(u)+\lambda_{d 2} f_{d 2}(u)}
$$

where

$$
\begin{aligned}
& \lambda_{n 0}=1+y^{2}, \lambda_{n 1}=1, \lambda_{n 2}=0.5 \\
& \lambda_{d 0}=e^{-y}+\cos (y)+y, \lambda_{d 1}=1
\end{aligned}
$$

And

$$
f_{n 1}(u)=u, f_{n 2}(u)=u^{3}, f_{d 1}(u)=u
$$

\subsection{U-State space model (Li et al., 2020)}

U-state space model, $U_{S S}$ in short is defined as below,

$$
U_{S S}:\left\{\begin{array}{l}
\dot{x}_{1}=\sum_{i=0}^{n} \lambda_{1 i} f_{1 i}\left(x_{2}\right) \\
\dot{x}_{2}=\sum_{i=0}^{n} \lambda_{2 i} f_{2 i}\left(x_{3}\right) \\
\dot{x}_{n}=\sum_{i=0}^{n} \lambda_{n i} f_{n i}(u) \\
y=\sum_{i=0}^{n} h_{i}\left(x_{1}, x_{2}, \ldots, x_{n}\right)
\end{array}\right.
$$


where $\lambda_{* *}$ is U-model time-varying parameters which can absorb all the other items except controller output vector $u$ and $f_{* *}$ is a smooth U-basis function.

Remark 4: U-state space model is a multi-layer U-polynormal since each line of $U_{S S}$ is $a U_{P}$.

Consider a simple non-linear state space model:

$$
\left\{\begin{array}{l}
\dot{x}_{1}=x_{1}+x_{1} x_{2} \\
\dot{x}_{2}=-x_{1}+u \\
y=x_{1}
\end{array}\right.
$$

Based on absorbing rule in equation (9), convert system (10) into U-state space model:

$$
\left\{\begin{array}{l}
\dot{x}_{1}=\lambda_{10}+\lambda_{11} f_{11}\left(x_{2}\right) \\
\dot{x}_{2}=\lambda_{20}+\lambda_{21} f_{21}(u) \\
y=x_{1}
\end{array}\right.
$$

where

$$
\begin{aligned}
& \lambda_{10}=x_{1}, \lambda_{11}=x_{1}, \lambda_{20}=-x_{1}, \lambda_{21}=1 \\
& f_{11}\left(x_{2}\right)=x_{2}, f_{21}(u)=u
\end{aligned}
$$

\subsection{U-control framework (Li et al., 2020)}

Assume the plant model $G_{P}$ is stable, bounded, and inversion exist. From Figure 1, there are two parts building the U-controller in the dashed line block area, these are, the invariant controller $G_{c 1}$ and U-dynamic inverter $G_{P}^{-1}$. Therefore, the expression of this $\mathrm{U}$-control system is:

$$
\sum=\left(U\left(G_{c 1}, G_{P}^{-1}\right) G_{p}\right)
$$

Figure 1 U-control system design framework

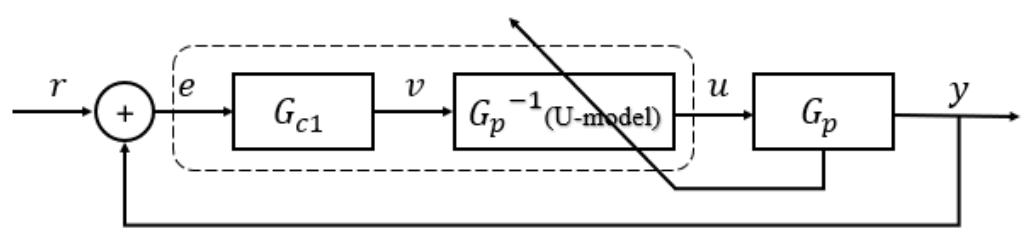

U-control system requires design $\mathrm{U}$-inverter $G_{P}^{-1}$ to make plant output $y$ equal to the invariant controller output $v$, that is, $v=y$, which means reaching $G_{P}^{-1} G_{P}=1$ under the perfect dynamic inversion. Therefore, U-control system performance depends on the 
accuracy of the plant dynamic inversion $G_{p}{ }^{-1}$, therefore, U-control is ineffective for controlling plants with inaccurate models or systems with disturbance.

Based on the U-control system expression in (13), if the plant is $G_{M}$ in practice, then U-control system is as shown in Figure 2.

Figure 2 U-control system framework with inaccurate plant

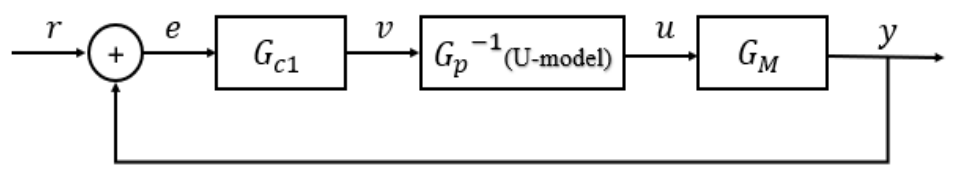

Let $k=\frac{G_{M}}{G_{p}}$, then the system input-output relationship is: $Y(s)=\frac{G_{c} k}{1+G_{c} k} U(s)$. In this case, system output $Y(s)$ will be impacted directly by the plant inaccuracy rate $k$. Then, consider an accurate system and disturbance $d(s)$ has been introduced in the system. The U-control system is as shown in Figure 3.

Figure 3 U-control system with uncertain plant and control disturbance

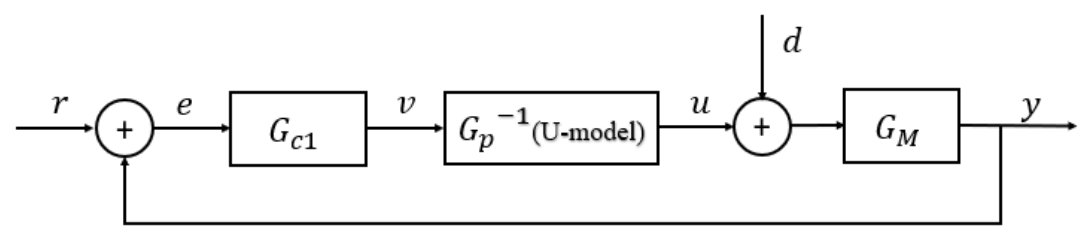

Accordingly, the system input-output relationship is: $Y(s)=\frac{G_{c} k}{1+G_{c} k} U(s)+\frac{G_{p} k}{1+G_{c} k} d(s)$, that is, the system output will be influenced by the system disturbance. In this case, system disturbance will be suppressed by the closed loop system, especially when $G_{c}$ is large. However, if the system has high frequency disturbance, this will not only affect the performance of the control system, but may also cause damage to the control system due to resonance. In summary, controlled plant inaccuracy and system disturbance will bring great challenges and difficulties to U-control system design.

\section{$3 \quad \mathrm{U}_{\mathrm{SM}}$-control framework and design procedure}

In Section 2, since these two main problems (plant inaccuracy and system disturbance) greatly degrade $\mathrm{U}$-control performance, this study proposes a new $\mathrm{U}_{\mathrm{SM}}$-control method which combines U-control and SMC to enhance the robustness of U-control. The $\mathrm{U}_{\mathrm{SM}^{-}}$-control system framework is shown in Figure 4. 
Figure $4 \mathrm{U}_{\mathrm{SM}^{-}}$-control system framework

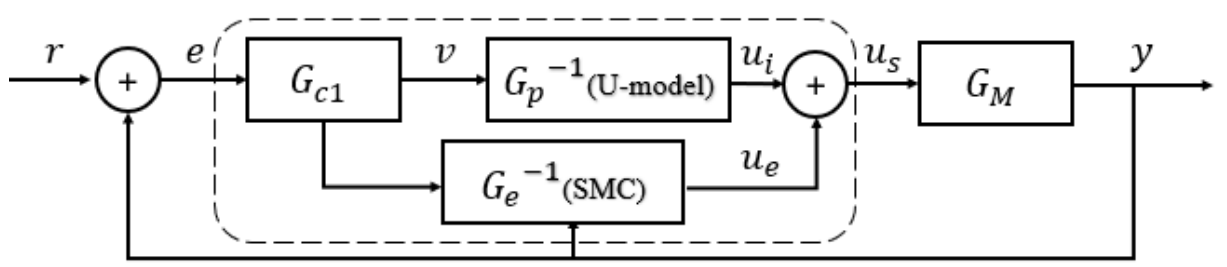

where $G_{M}$ is the controlled plant model in practice which may contain uncertain system coefficients, $r$ is the desired output, which is also called reference in control system, $y$ is the system output and $e$ is the difference between $y$ and $r$ in this closed-loop control system. $G_{c 1}$ is U-model-based linear invariant controller, which can be designed by users for different control performance. $G_{e}^{-1}(\mathrm{SMC})$ model is the core part in the $\mathrm{U}_{\mathrm{SM}}$-control system, which can compensate the inversion error of the plant model $G_{M}$. Similar to U-control, $\mathrm{U}_{\mathrm{SM}}$-control framework requests dynamic inverse $G_{P}^{-1}$ exist. In general, the $\mathrm{U}_{\mathrm{SM}}$-control system design procedure has three separate steps:

1) Design ideal plant inverter $G_{P}^{-1}$ : Based on U-control system design framework in Sub-section 2.4, replace ideal plant model $G_{P}$ in the U-control system with the actual plant model $G_{M}$. Accordingly, system (13) can be converted into:

$$
\sum=\left(U\left(G_{c 1}, G_{P}^{-1}\right), G_{M}\right)
$$

2) Design compensated plant inverter $G_{e}^{-1}$ : From Sub-section 2.4 , it is clear that system (14) will be influenced by system mismatch. Assume the practice plant model $G_{M}$ is bounded, and its ideal inverse $G_{P}^{-1}$ exists. From Figure 3, the $\mathrm{U}_{\mathrm{SM}}$-controller in the dashed line block has three parts: the invariant controller $G_{c 1}$, the ideal plant's dynamic inverter $G_{P}^{-1}$ and the compensated plant inversion $G_{e}^{-1}$. To facilitate the design of $G_{e}^{-1}$, system (14) can be converted into:

$$
\sum=\left(U\left\{G_{c 1},\left(G_{P}^{-1}, G_{e}^{-1}\right)\right\}, G_{M}\right)
$$

where $\left\{G_{c 1},\left(G_{P}^{-1}, G_{e}^{-1}\right)\right\}$ is defined as $\mathrm{U}_{\mathrm{SM}}$-controller. Same as the operating principle in the U-control system, $\mathrm{U}_{\mathrm{SM}}$-inverter $\left(G_{P}^{-1}+G_{e}^{-1}\right)$ is designed to force and ensure the plant output $y$ equals to the invariant controller output $v$.

3) Design U-control invariant controller $G_{c 1}$ : By assuming $\left(G_{P}^{-1}+G_{e}^{-1}\right) G_{M}=1$, Figure 4 is therefore simplified to Figure 5 and gives:

$$
\sum=\left(G_{c 1}, 1\right)
$$


Equation (16) shows a typical classical linear control system. Assume the gain of the closed-loop control system is $G$ and therefore from equation (16), $G$ can be expressed as $G=\frac{G_{c 1}}{1+G_{c 1}}$. Generally, $G$ can be specified and designed with different requirements by users, typically different damping ratio and natural frequency $\omega_{n}$ can reach different control performance. In this case, the invariant controller $G_{c 1}$ in U-control system can be calculated based on different system closed-loop gain by: $G_{c 1}=\frac{G}{1-G}$.

Figure 5 Simplified $\mathrm{U}_{\mathrm{SM}}$-control system

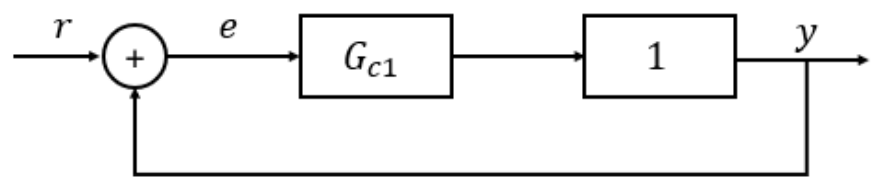

\section{Design of plant dynamic inverter $G_{p}^{-1}$ and compensated plant inverter $G_{e}^{-1}$}

From the $\mathrm{U}_{\mathrm{SM}}$-control system design framework, $\mathrm{U}_{\mathrm{SM}}$-controller contains two parts: ideal plant inverter $G_{p}^{-1}$ and compensated plant inverter $G_{e}^{-1}$ and this study defines their output as $u_{i}$ and $u_{e}$, respectively.

Consider a general underactuated SISO CT state space model:

$$
\left\{\begin{array}{l}
\dot{X}=F(X, u) \\
y=H(X)
\end{array}\right.
$$

where $u, y \in \mathbb{R}$ are plant input and output, respectively, $X \in \mathbb{R}^{n}$ represents all state variables. $F \in \mathbb{R}$ introduces the relationship between state variables and plant input, $H \in \mathbb{R}$ is a smooth mapping to drive all state variables to the plant output. Expand this multi-layer model (17) into a multi-layer polynormal expression as follows:

$$
\left\{\begin{array}{l}
\dot{x}_{1}=F_{1}\left(x_{1}, x_{2}, \ldots, x_{n-1}, x_{n}\right) \\
\dot{x}_{2}=F_{2}\left(x_{1}, x_{2}, \ldots, x_{n-1}, x_{n}\right) \\
\vdots \\
\dot{x}_{n-1}=F_{2}\left(x_{1}, x_{2}, \ldots, x_{n-1}, x_{n}\right) \\
\dot{x}_{n}=F_{n}\left(x_{1}, x_{2}, \ldots, x_{n-1}, x_{n}\right)+g\left(x_{1}, x_{2}, \ldots, x_{n}\right) u+d \\
y=x_{1}
\end{array}\right.
$$

In system (18), $F_{*} \in \mathbb{R}^{n}$ is a smooth function absorbing all state variables $x_{i}, g$ is a function to represent the relationship between state variables and controller output $u, d$ 
is the system interference/error and $|d|<\bar{d}$. For the next work in this study, assuming that there is no unstable zero dynamics in controlled system and all the state variables $x_{i}$ can be observed.

\subsection{Design of ideal plant inverter $\boldsymbol{G}_{\boldsymbol{p}}^{-1}$}

Ignore control interference $d$ and convert state space model (18) into a multi-layer U-model expression (12) as follows:

$$
\left\{\begin{array}{l}
\dot{x}_{1}=\sum_{i=1}^{n} \lambda_{1 i} f_{1 i}\left(x_{i}\right) \\
\dot{x}_{2}=\sum_{i=1}^{n} \lambda_{2 i} f_{2 i}\left(x_{i}\right) \\
\vdots \\
\dot{x}_{n}=\sum_{i=1}^{n} \lambda_{n i} f_{n i}\left(x_{i}\right)+u_{i} \\
y=x_{1}
\end{array}\right.
$$

Differentiate $y$ in (19) and gives:

$$
\dot{y}=\dot{x}_{1}=\sum_{i=1}^{n} \lambda_{1, i} f_{1, i}\left(x_{i}\right)
$$

If equation (20) cannot show the direct relationship between $\dot{y}$ and $u$, then differentiate $\dot{y}$ and replace $\dot{x}_{i}$ items in system (20) with the polynomial expression for each $\dot{x}_{i}$ in (19). Repeat this procedure to find the direct relationship between $\frac{(m)}{y}$ and $u$, where $(m)$ is the $m$ times derivative of system output $y$. After organisation, it gives: $y$

$$
\stackrel{(m)}{y}=\lambda_{1}\left(x_{1}, x_{2}, \ldots, x_{n-1}, x_{n}\right)+\lambda_{2}\left(x_{1}, x_{2}, \ldots, x_{n-1}, x_{n}\right) u_{i}
$$

where $\lambda_{i}$ is a time-varying parameter vector function absorbing all state variables. It is clear that equation (21) is a simplified form of general U-model polynomial expression (1). Therefore, the ideal inverter output $u_{i}$ is:

$$
u_{i}=\frac{(j)-\lambda_{1}\left(x_{1}, x_{2}, \ldots, x_{n-1}, x_{n}\right)}{\lambda_{2}\left(x_{1}, x_{2}, \ldots, x_{n-1}, x_{n}\right)}
$$


Additionally, state variables $x_{i}$ can be obtained by integrating $\dot{x}_{i}$ in the feedback loop from the plant, that is, $x_{i}=\dot{x}_{i} \frac{1}{S}$, where without confusion, $\frac{1}{S}$ is the integral operation of Laplace transform.

\subsection{Design of compensated plant inverter $\boldsymbol{G}_{e}^{-1}$}

This study introduces Sliding Mode Control (SMC) into U-control to strengthen robustness, in way of adding compensated plant inverter $G_{e}^{-1}$ to cope with plant uncertainties and system disturbances. For determining $G_{e}^{-1}$, define the $m$-th order of state error equations as:

$$
\left\{\begin{array}{l}
e_{1}=x_{1}-x_{d} \\
e_{2}=e_{1}=x_{1}-\dot{x}_{d} \\
e_{3}=e_{2}=x_{1}-x_{d} \\
\vdots \\
e_{m}=\dot{e}_{m-1}=\begin{array}{c}
(m-1) \\
x_{1}
\end{array}-\begin{array}{c}
(m-1) \\
x_{d}
\end{array}
\end{array}\right.
$$

where $\begin{gathered}(m-1) \\ x_{1}\end{gathered}$ and $\begin{gathered}(m-1) \\ x_{d}\end{gathered}$ are the $(m-1)-t h$ order derivatives of the plant output $x_{1}$ and desired output $x_{d}$. Then, define a sliding surface function $\sigma$ (Shtessel, 2014) by:

$$
\sigma=c_{1} e_{1}+c_{2} e_{2}+\ldots+c_{m-1} e_{m-1}+e_{m}
$$

From equation (24), the derivative of the sliding surface function is

$$
=c_{1}\left(\dot{x}_{1}-x_{d}\right)+c_{2}\left(\dot{x}_{2}-x_{d}\right)+\ldots+c_{n-1}\left(\begin{array}{c}
(m-1) \\
x_{1}-\frac{(m-1)}{x_{d}}
\end{array}\right)+\left(\begin{array}{c}
(m)-(m) \\
x_{1}
\end{array}\right)
$$

where $c_{i} \in R^{+}, i \in R^{+}, \quad c_{i}$ is the coefficient specifying the bandwidth of the sliding surface function (Shtessel, 2014).

Same as the U-model state space expression, replace the highest order derivative of the desired output $\begin{gathered}(m) \\ x_{d}\end{gathered}$ with $v$, in this case, the other orders derivatives of the desired output ${ }_{x_{d}}^{(i)}(0<i<m)$ can be expressed by the $(m-i)$ order integration of $v$, that is, $\begin{aligned} & (i) \\ & x_{d}\end{aligned}=v\left(\frac{1}{s}\right)^{m-i}$, where $\frac{1}{s}$ is the Laplace transform of integration. In this case, error equations (23) and (25) can be converted into: 


$$
\begin{aligned}
& e_{1}=x_{1}-v\left(\frac{1}{s}\right)^{m} \\
& e_{2}=e_{1}=x_{1}-v\left(\frac{1}{s}\right)^{m-1} \\
& e_{3}=e_{2}=x_{1}-v\left(\frac{1}{s}\right)^{m-2} \\
& \vdots \\
& e_{m}=\dot{e}_{m-1}=\frac{(m-1)}{x_{1}}-v\left(\frac{1}{s}\right) \\
& \dot{\sigma}=c_{1}\left(\dot{x}_{1}-v\left(\frac{1}{s}\right)^{m-1}\right)+c_{2}\left(x_{2}-\left(\frac{1}{s}\right)^{m-2}\right)+\ldots+\left(\begin{array}{c}
(m) \\
x_{1}
\end{array}\right) \\
& =\sum_{i=1}^{m-1}\left(\begin{array}{c}
(i) \\
c_{i} \\
\\
x_{1}
\end{array}-v\left(\frac{1}{s}\right)^{m-i}\right)+\left(\begin{array}{c}
(m)-v \\
x_{1}
\end{array}\right)
\end{aligned}
$$

In the actual calculation process, direct calculation procedure of $\underset{x_{1}}{(m)}$ can be converted as continuously differentiating the lower order derivatives of $\frac{(i)}{x_{1}}$ and replace its first order derivates items with corresponding items in equation (26) until $i$ equals $m$. The first $(m-2)$ times differentiating operation does not have $x_{n}$ items, therefore, the expression of a series of derivatives of $x_{1}$ are:

$$
\left\{\begin{array}{l}
\dot{x}_{1}=F_{1}\left(x_{1}, x_{2}, \ldots, x_{n-1}\right)=\mathcal{F}_{1}\left(x_{1}, x_{2}, \ldots, x_{n-1}\right) \\
x_{1}=\dot{\mathcal{F}}_{1}=\frac{\partial \mathcal{F}_{1}}{\partial x_{1}} F_{1}+\frac{\partial \mathcal{F}_{1}}{\partial x_{2}} F_{2}+\ldots+\frac{\partial \mathcal{F}_{1}}{\partial x_{n-1}} F_{n-1}=\mathcal{F}_{2}\left(x_{1}, x_{2}, \ldots, x_{n-1}\right) \\
\dddot{x}_{1}=\dot{\mathcal{F}}_{2}=\frac{\partial \mathcal{F}_{2}}{\partial x_{1}} F_{1}+\frac{\partial \mathcal{F}_{2}}{\partial x_{2}} F_{2}+\ldots+\frac{\partial \mathcal{F}_{2}}{\partial x_{n-1}} F_{n-1}=\mathcal{F}_{3}\left(x_{1}, x_{2}, \ldots, x_{n-1}\right) \\
\vdots \\
(m-1)=\dot{\mathcal{F}}_{m-2}=\frac{\partial \mathcal{F}_{m-2}}{\partial x_{1}} F_{1}+\frac{\partial \mathcal{F}_{m-2}}{\partial x_{2}} F_{2}+\ldots+\frac{\partial \mathcal{F}_{m-2}}{\partial x_{n-1}} F_{n-1}=\mathcal{F}_{m-1}\left(x_{1}, x_{2}, \ldots, x_{n-1}, x_{n}\right) \\
x_{1} \\
(m)=\frac{\partial \mathcal{F}_{m-1}}{\partial x_{1}} F_{1}+\frac{\partial \mathcal{F}_{m-1}}{\partial x_{2}} F_{2}+\ldots+\frac{\partial \mathcal{F}_{m-1}}{\partial x_{n-1}} F_{n-1}+\frac{\partial \mathcal{F}_{m-1}}{\partial x_{n}}\left(F_{n}+g u_{s}+d\right) \\
x_{1}
\end{array}\right.
$$

In this case, equation (27) can be expanded to: 


$$
\begin{aligned}
& \dot{\sigma}=\sum_{i=1}^{m-1}\left(\begin{array}{c}
(i) \\
c_{i} \\
\\
x_{1}
\end{array}-v\left(\frac{1}{s}\right)^{m-i}\right)+\left(\begin{array}{c}
(m)-v \\
x_{1}
\end{array}\right) \\
& =\sum_{i=1}^{m-1} c_{i}\left(\mathcal{F}_{i}-v\left(\frac{1}{s}\right)^{m-i}\right) \\
& +\left(\frac{\partial \mathcal{F}_{m-1}}{\partial x_{1}} F_{1}+\frac{\partial \mathcal{F}_{m-1}}{\partial x_{2}} F_{2}+\ldots+\frac{\partial \mathcal{F}_{m-1}}{\partial x_{n-1}} F_{n-1}+\frac{\partial \mathcal{F}_{m-1}}{\partial x_{n}}\left(F_{n}+g u+d\right)-v\right)
\end{aligned}
$$

Let $\dot{\sigma}=0$, to obtain the equivalent controller output and switching controller output (under exponential approach law) (Shtessel, 2014) by

$$
\begin{aligned}
& u_{e q}=-\left[\frac{\partial \mathcal{F}_{m-1}}{\partial x_{n}} g\right]^{-1}\left\{\sum_{i=1}^{m-1} c_{i}\left(\mathcal{F}_{i}-v\left(\frac{1}{s}\right)^{m-i}\right)+\left(\frac{\partial \mathcal{F}_{m-1}}{\partial x_{1}} F_{1}+\frac{\partial \mathcal{F}_{m-1}}{\partial x_{2}} F_{2}+\ldots+\frac{\partial \mathcal{F}_{m-1}}{\partial x_{n}} F_{n}-v\right)\right\} \\
& u_{s w}=-\left[\frac{\partial \mathcal{F}_{m-1}}{\partial x_{n}} g\right]^{-1}[\operatorname{ssgn}(\sigma)+k \sigma], \varepsilon>0, k>0
\end{aligned}
$$

From equation (22) and $\mathrm{U}_{\mathrm{SM}}$-control system framework (14), except invariable controller, $\mathrm{U}_{\mathrm{SM}}$-controller should contain two parts: the ideal inverter output $u_{i}$ and the compensated inverter output $u_{c}$. Therefore, $\mathrm{U}_{\mathrm{SM}}$-controller output $u_{s}=u_{e q}+u_{s w}$ $=u_{i}+u_{c}$, that is, $u_{c}=\left(u_{e q}-u_{i}\right)+u_{s w}$. Then, bring $\mathrm{U}_{\mathrm{SM}}$-controller output $u_{s}$ into equation (29):

$$
\dot{\sigma}=-\varepsilon \operatorname{sgn}(\sigma)-k \sigma+\frac{\partial \mathcal{F}_{m-1}}{\partial x_{n}} d
$$

Let $\varepsilon=\bar{d}+\rho, \rho>0$. The Lyapunov function can be defined as $V=\frac{1}{2} \sigma^{2}$, then:

$$
\begin{aligned}
\dot{V} & =\sigma \dot{\sigma}=\sigma\left(-(\bar{d}+\rho) \operatorname{sgn}(\sigma)-k \sigma+\frac{\partial \mathcal{F}_{m-1}}{\partial x_{n}} d\right) \\
& =-(\bar{d}+\rho)|\sigma|-k \sigma^{2}+\sigma \frac{\partial \mathcal{F}_{m-1}}{\partial x_{n}} d \leq-\rho|\sigma|-k \sigma^{2} \leq 0
\end{aligned}
$$

\section{Case studies}

In this part, a simplified helicopter pitch dynamics model is selected to test the $\mathrm{U}_{\mathrm{SM}}$-control method. Firstly, Sub-section 5.1 introduces this physical model for the operation and its state space model for control system design. Then, $\mathrm{U}_{\mathrm{SM}}$-controller which includes invariable controller $G_{c 1}$, ideal plant inverter $G_{P}^{-1}$ and compensated plant inverter $G_{e}^{-1}$ will be designed step by step from Sub-section 5.2 to Sub-section 5.4. Subsection 5.5 demonstrates the consistency of $\mathrm{U}_{\mathrm{SM}}$-control method and U-control method 
under the ideal error-free model. Sub-section 5.6 tests the robustness of proposed $\mathrm{U}_{\mathrm{SM}}$ control method. Sub-section 5.6.1 considers a simplified helicopter pitch dynamics model with system internal uncertainty and Sub-section 5.6.2 introduces system noise based on the previous uncertain plant. Then, by comparing the simulation results, the robustness of $\mathrm{U}_{\mathrm{SM}}$-control method is verified. Finally, Sub-section 5.7 discusses all simulation results and gives a brief conclusion.

\subsection{Simplified helicopter pitch dynamic model}

The schematic diagram of the helicopter (Razavi et al., 2016; Samadi and Rodrigues, 2009 ) is shown in Figure 6. The non-linear model of the pitch dynamics of the simplified helicopter is described by the following equation:

$$
\ddot{\theta} I_{y y}+m_{h e l} g l_{c g x} \cos \theta+m_{h e l} g l_{c g z} \sin \vartheta+F_{v M} \dot{\theta}
$$

This simplified helicopter model has two degrees of freedom in vertical direction with only one actuator: propeller lifting power. Let $\theta=x_{1}, \dot{\theta}=x_{2}$, convert non-linear system (33) into state space expression:

$$
\left\{\begin{array}{l}
\dot{x}_{1}=x_{2} \\
\dot{x}_{2}=\frac{1}{I_{y y}}\left(-m_{h e l} l_{c g x} g \cos \left(x_{1}\right)-m_{h e l} l_{c g z} g \sin \left(x_{1}\right)-F_{v M} x_{2}+u\right)
\end{array}\right.
$$

where $x_{1}$ is the pitch angle $\theta$ and $x_{2}$ represents the pitch rate $\omega ; I_{y y}$ is the second moment around the $y$-axis; $m_{h e l}$ is the mass of the helicopter; $l_{c g x}$ and $l_{c g z}$ are displacements from the centre point of mass (GC in Figure 6) relative to the rotation joint $B$ shown in Figure 6; $F_{v M}$ is the pitch damping; $u$ is the control torque exerted by the main blade of the helicopter around the $y$-axis;

\subsection{Invariant controller $\boldsymbol{G}_{c 1}$ design}

In order to ensure the system has no overshoot, therefore, system (34) should be a critical damping system which returns the system to equilibrium (desired reference) as fast as possible without overshooting. With reference to $\mathrm{U}_{S M}$-control system design procedure proposed in Section 3, this study chooses system damping ratio 1 and natural frequency $\omega_{n}=2$. Accordingly, the desired closed-loop system gain is assigned with:

$$
\frac{Y(S)}{R(S)}=G(S)=\frac{4}{s^{2}+4 s+4}
$$

From Sub-section 2.4, the invariant controller $G_{c 1}$ with a unit constant plant in a feedback control system is determined by taking inverse of the closed loop transfer function (35) as below:

$$
G_{c 1}=\frac{G}{1-G}=\frac{4}{s^{2}+4 s}
$$


Figure 6 Simplified pitch model of the helicopter (Razavi et al., 2016)

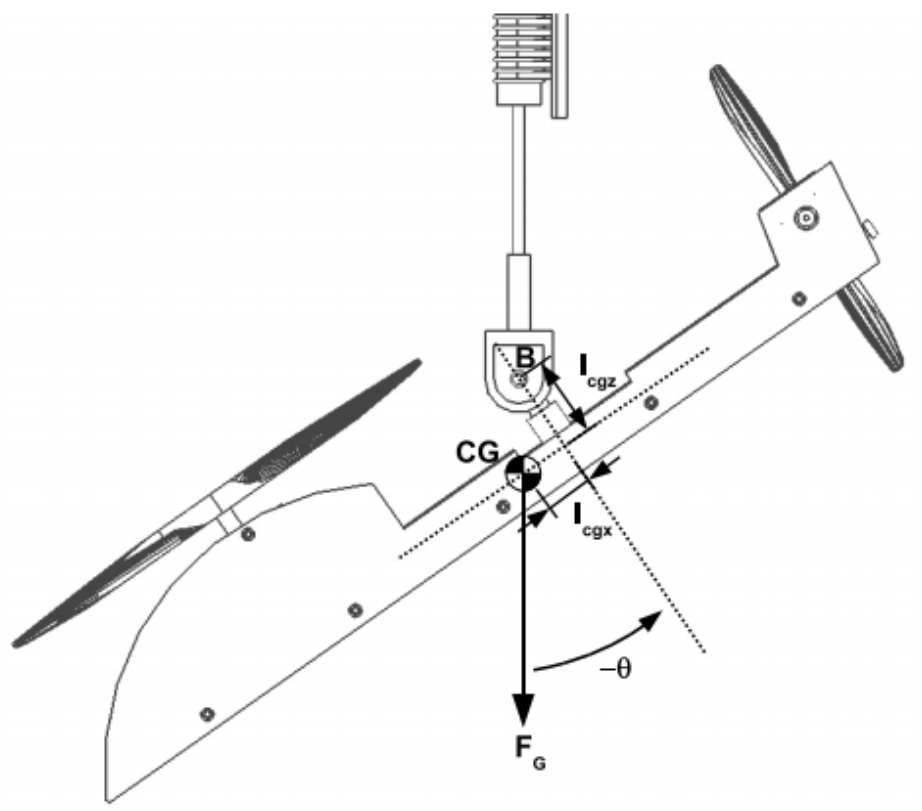

\subsection{Ideal inverter $\boldsymbol{G}_{\boldsymbol{P}}^{-1}$ design}

Sub-section (4.1) introduces ideal plant dynamic inverter $G_{P}^{-1}$ design procedure.

Continue take the derivative to $y$ and replace $\dot{x}_{i}(i>0)$ with equation in (34). Ignore noise item, then it gives:

$$
\ddot{y}=\dot{x}_{2}=\frac{1}{I_{y y}}\left(-m_{h e l} l_{c g x} g \cos \left(x_{1}\right)-m_{h e l} l_{c g z} g \sin \left(x_{1}\right)-F_{v M} x_{2}+u\right)
$$

Therefore, the original system is converted into U-model expression:

$$
\left\{\begin{array}{l}
\ddot{y}=\lambda_{0}+\lambda_{1} u \\
\lambda_{0}=\frac{1}{I_{y y}}\left(-m_{h e l} l_{c g x} g \cos \left(x_{1}\right)-m_{h e l} l_{c g z} g \sin \left(x_{1}\right)-F_{v M} x_{2}\right) \\
\lambda_{1}=\frac{1}{I_{y y}}
\end{array}\right.
$$

Then, the inverter $G_{P}^{-1}$ output is designed as:

$$
u_{i}=\frac{y-\lambda_{0}}{\lambda_{1}}
$$


Let $\bar{y}$ be replaced with $v$, then:

$$
u_{i}=\frac{v-\lambda_{0}}{\lambda_{1}}
$$

\subsection{Compensated inverter $\boldsymbol{G}_{\boldsymbol{e}}^{-1}$ design}

Based on Sub-section (4.2), the error equations are designed as:

$$
\left\{\begin{array}{l}
e_{1}=x_{1}-v\left(\frac{1}{s}\right)^{2} \\
e_{2}=e_{1}=\dot{x}_{1}-v \frac{1}{s}
\end{array}\right.
$$

The sliding function (Shtessel, 2014) is:

$$
\sigma=c e_{1}+e_{2}
$$

From equations (42) and (43), we have:

$$
\begin{aligned}
\dot{\sigma} & =c e_{1}+e_{2}=c\left(\dot{x}_{1}-v \frac{1}{s}\right)+\left(\dot{x}_{2}-v\right) \\
& =c\left(x_{2}-v \frac{1}{s}\right)+\left(\frac{1}{I_{y y}}\left(-m_{h e l} l_{c g x} g \cos \left(x_{1}\right)-m_{h e l} l_{c g z} g \sin \left(x_{1}\right)-F_{v M} x_{2}\right)+\frac{u}{I_{y y}}-v\right)
\end{aligned}
$$

Bring U-model expression equation (39) into (44), equation (44) can be converted into:

$$
\begin{aligned}
& \dot{\sigma}=c\left(x_{2}-v \frac{1}{s}\right)+\left(\lambda_{0}+\lambda_{1} u-v\right) \\
& u_{e q}=\frac{v-c\left(x_{2}-v \frac{1}{s}\right)-\lambda_{0}}{\lambda_{1}} \\
& u_{s w}=-(\operatorname{sggn}(\sigma)+k \sigma)
\end{aligned}
$$

Based on equation (45), $\varepsilon=\bar{d}+\rho, \rho>0$. There is no disturbance first, let $\rho=0.1$ and $\varepsilon=\bar{d}+\rho=0.1$. In order to keep the sliding rate (Shtessel, 2014), let $k=1$, then the output of the compensated inverter $G_{e}^{-1}$ is:

$$
\begin{aligned}
u_{s}= & u_{e q}+u_{s w}-u_{i} \\
= & \frac{v-10\left(x_{2}-v \frac{1}{s}\right)-\lambda_{0}}{\lambda_{1}}-\frac{v-\lambda_{0}}{\lambda_{1}}-(\operatorname{sgn}(\sigma)+\sigma) \\
= & \frac{-10\left(x_{2}-v \frac{1}{s}\right)}{\lambda_{1}}-(\operatorname{sgn}(\sigma)+\sigma)
\end{aligned}
$$


To avoid system chattering, the switching operation should not be hold if the system dynamic sliding inside the boundary $\xi \in R^{+}$. When the system dynamic is outside the boundary $\xi$, the switching function is defined by:

$$
\operatorname{sat}(\sigma)=\left\{\begin{array}{cc}
\operatorname{sgn}(\sigma), & |\sigma| \geq \xi \\
\frac{\sigma}{\xi}, & |\sigma|<\xi
\end{array}\right.
$$

For more precise convergence and because this system has no disturbance or uncertainty, $\xi$ should be small. Let $\xi=0.05$, then the compensated inverter $G_{e}^{-1}$ is shown:

$$
u_{s}=\frac{-10\left(x_{2}-v \frac{1}{s}\right)}{\lambda_{1}}-(\operatorname{sat}(\sigma)+\sigma)
$$

where $\lambda_{1}=\frac{1}{I_{y y}}$.

\subsection{Perfectly matched plant simulation test}

Based on Razavi et al. (2016), the experiment modelling parameters are shown in Table 1.

Table 1 Pitch model of the helicopter characteristics

\begin{tabular}{ccc}
\hline Parameter & Value & Unit \\
\hline$I_{y y}$ & 0.0283 & $\mathrm{kgm}^{2}$ \\
$m_{h e l}$ & 0.9941 & $\mathrm{~kg}$ \\
$l_{c g x}$ & 0.0134 & $\mathrm{~m}$ \\
$l_{c g z}$ & 0.0289 & $\mathrm{~m}$ \\
$F_{v M}$ & 0.0041 & $\frac{\mathrm{Nm}}{\mathrm{rad}}$ \\
$g$ & 9.81 & $\mathrm{~s}$ \\
\hline
\end{tabular}

For U-model (39), it is clear that U-model-based time varying parameters are:

$$
\left\{\begin{array}{l}
\ddot{y}=\lambda_{0}+\lambda_{1} u \\
\lambda_{0}=-4.618 \cos \left(x_{1}\right)-9.958 \sin \left(x_{1}\right)-0.1449 x_{2} \\
\lambda_{1}=\frac{1}{0.0283}
\end{array}\right.
$$


The framework of the ideal plant system applied with $\mathrm{U}_{\mathrm{SM}^{-}}$-control method is shown in Figure 7. Figure 8 shows the comparison results of ideal simplified helicopter model controlled by $\mathrm{U}$-control method and $\mathrm{U}_{\mathrm{SM}}$-control method. Figure 9 shows the compensated inverter output.

Figure $7 \quad \mathrm{U}_{\mathrm{SM}^{-}}$-control of helicopter

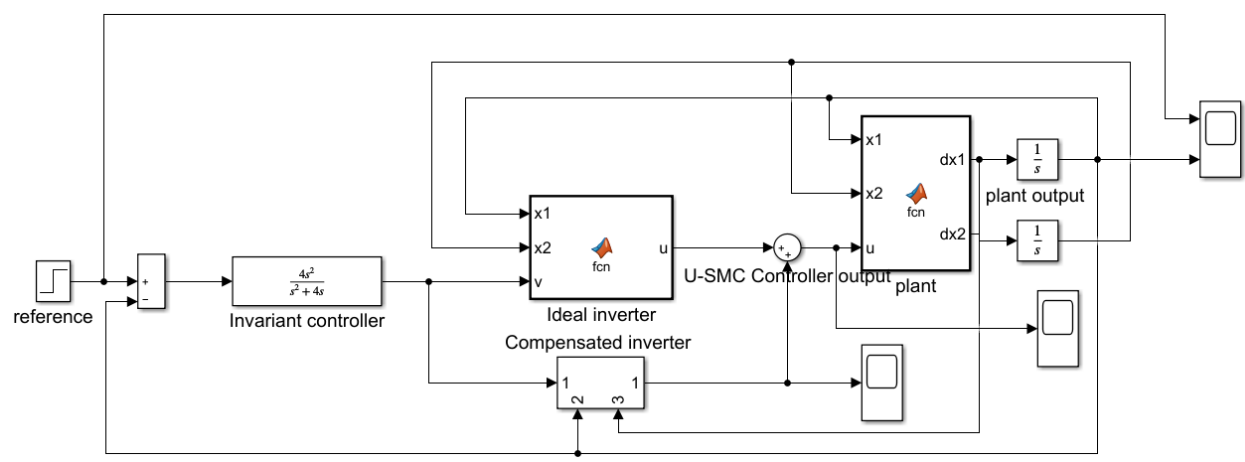

Figure 8 Comparison of perfectly matched simplified helicopter model. (a) $\mathrm{U}_{\mathrm{SM}^{-}}$-control-based plant output and reference (b) U-control-based plant output and reference (c) $\mathrm{U}_{\mathrm{SM}^{-}}$-controller output (d) U-controller output

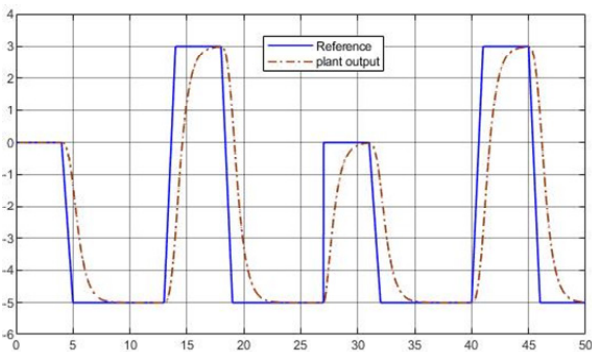

(a)

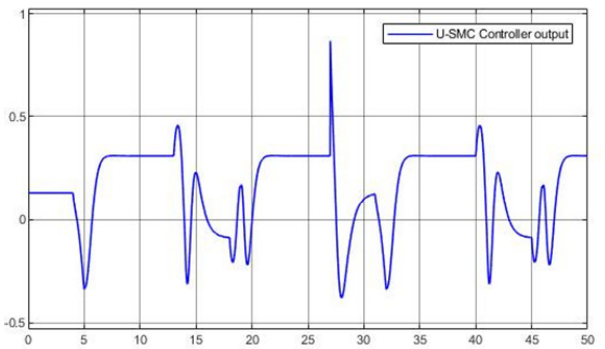

(c)

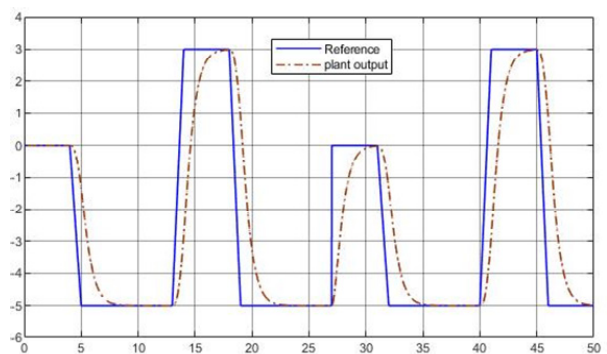

(b)

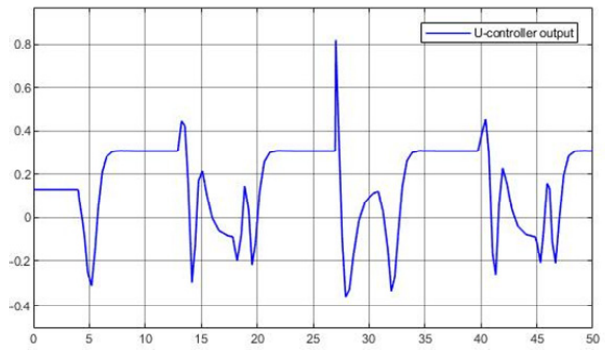

(d) 
Figure $9 \mathrm{U}_{\mathrm{SM}^{-}}$-control-based compensated inverter output

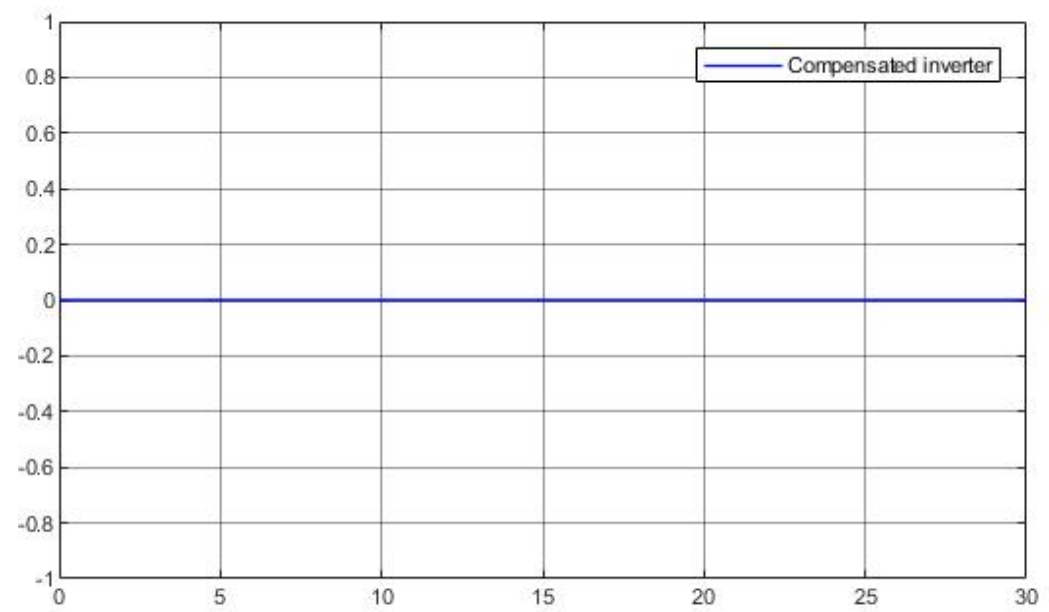

\subsection{Robustness testing and comparison}

\subsubsection{Plant with internal uncertainty}

In practical control system operation, a close-loop system output may be not measured accurately and is subject to the model imperfectly match. Therefore, to accommodate such inaccuracies, system (34) is changed into:

$$
\dot{x}_{1}=\Phi x_{2}\left\{\begin{array}{l}
\dot{x}_{1}=\Phi x_{2} \\
\dot{x}_{2}=\frac{1}{I_{y y}}\left(-m_{h e l} l_{c g x} g \cos \left(x_{1}\right)-m_{h e l} l_{c g z} g \sin \left(x_{1}\right)-F_{v M} x_{2}+u\right)
\end{array}\right.
$$

where $\Phi$ is the system uncertain coefficient which the amplitude changes from 0.5 to 1 with rate of $1 \mathrm{~Hz}$. Figure 10 shows its variation curve.

Figure 10 System uncertain coefficient

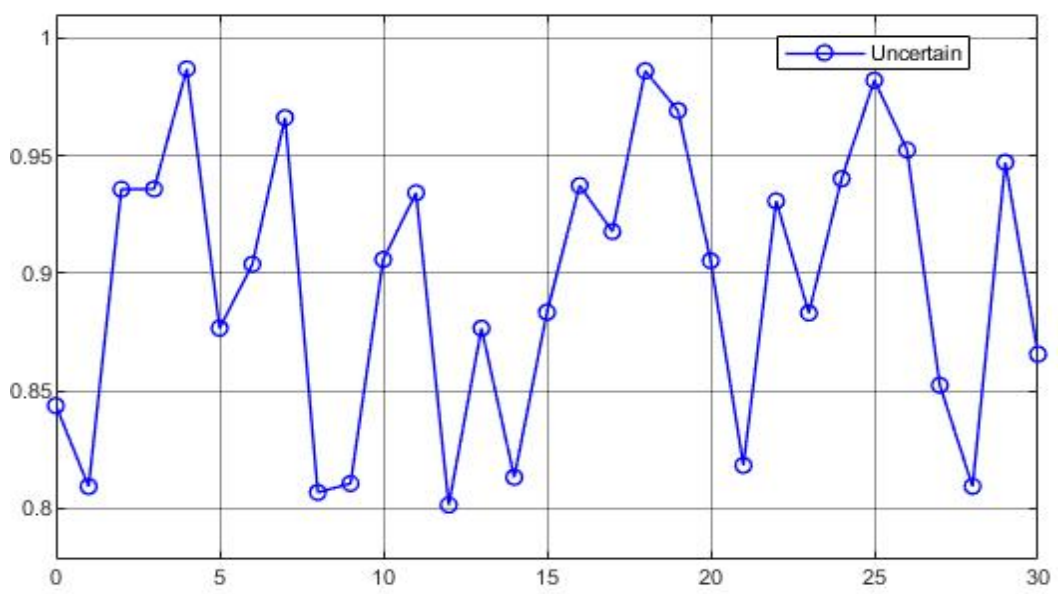


Remark 8: $\Phi$ is randomly changed within its boundary, that is, the uncertain system will deviate by up to 50\% from the perfectly matched system. To avoid chattering, $\xi$ should be larger than before. Let $\xi=0.1$, then the switching function is shown:

$$
\operatorname{sat}(\sigma)=\left\{\begin{array}{cc}
\operatorname{sgn}(\sigma), & |\sigma| \geq 0.1 \\
10 \sigma, & |\sigma|<0.1
\end{array}\right.
$$

Figure 11 shows the framework of $\mathrm{U}_{\mathrm{SM}^{-}}$-control system for plant with system uncertainty. Figure 12 shows the controlled comparison results of the simplified helicopter model with internal uncertain parameter by $U$-control method and $U_{\mathrm{SM}}$-control method. Figure 13 shows the output of the compensated inverter.

Figure $11 \mathrm{U}_{\mathrm{SM}^{-}}$-control of helicopter system uncertainty

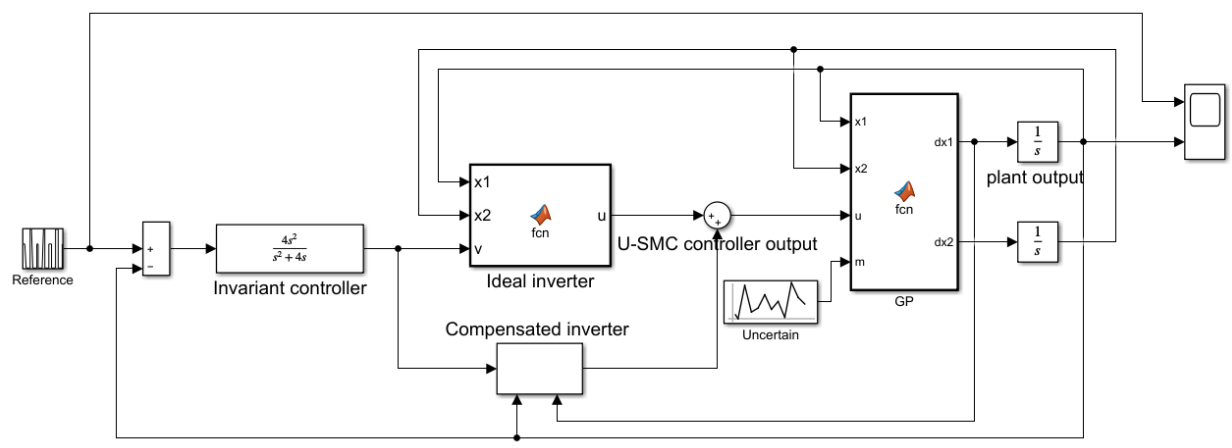

Figure 12 Comparison of mismatched simplified helicopter model. (a) $\mathrm{U}_{\mathrm{SM}^{-}}$-control-based plant output and reference (b) U-control-based plant output and reference (c) $\mathrm{U}_{\mathrm{SM}^{-}}$-controller output (d) U-controller output

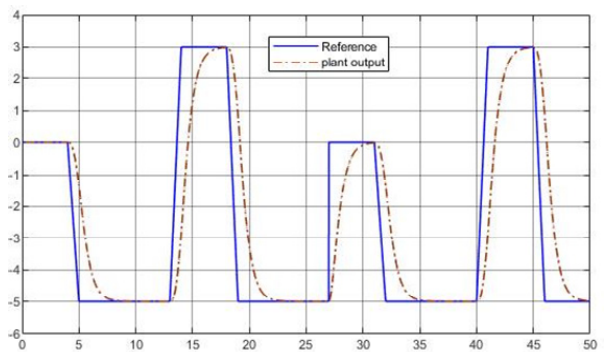

(a)

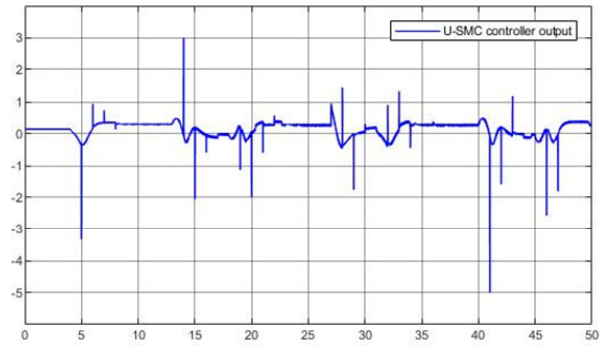

(c)

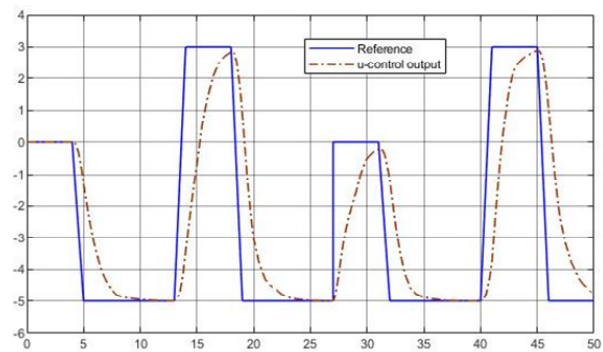

(b)

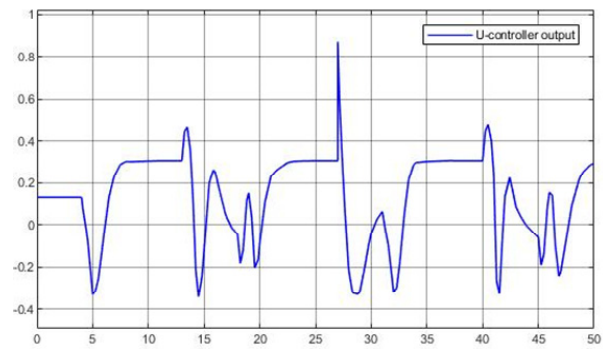

(d) 
Figure $13 \mathrm{U}_{\mathrm{SM}^{-}}$-control-based compensated inverter output

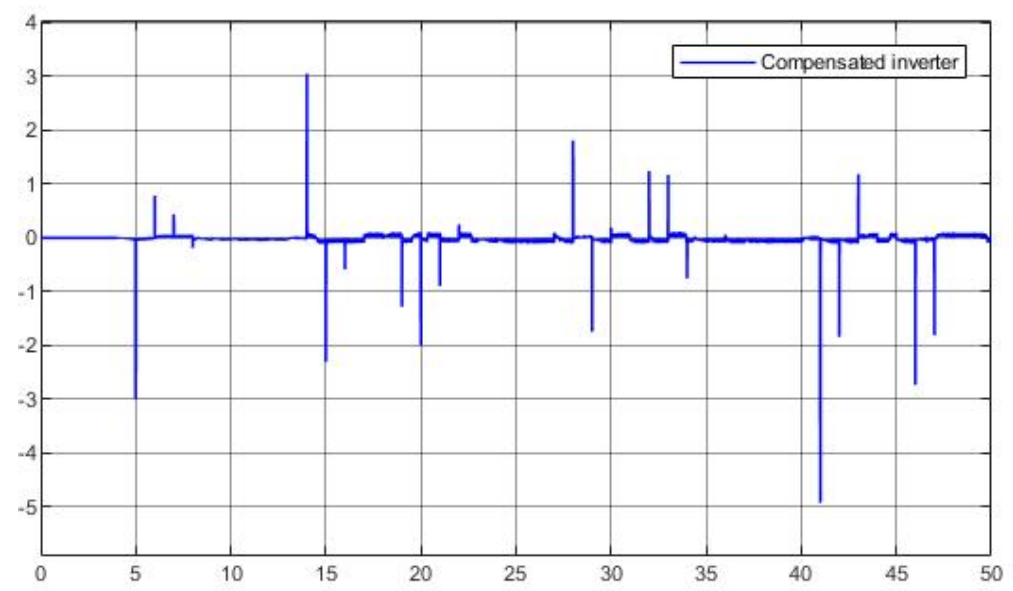

\subsubsection{Uncertain plant with system noise}

Consider a more complex and practical control situation: there are system internal uncertainty during the modelling process and system noise at the interface between the controller and the controlled platform. Therefore, to accommodate such inaccuracies, system (52) is changed into:

$$
\left\{\begin{array}{l}
\dot{x}_{1}=\Phi x_{2} \\
\dot{x}_{2}=\frac{1}{I_{y y}}\left(-m_{h e l} l_{c g x} g \cos \left(x_{1}\right)-m_{h e l} l_{c g z} g \sin \left(x_{1}\right)-F_{v M} x_{2}+u\right)+d
\end{array}\right.
$$

where $d$ is system noise/disturbance. $\Phi$ changes the same as it in system (53) from 0.5 to 1 with $1 \mathrm{~Hz}$ variation frequency. $d$ changes from 0 to 0.3 with $10 \mathrm{~Hz}$. Figure 14 shows system noise/disturbance variation curve.

Figure 14 System control noise

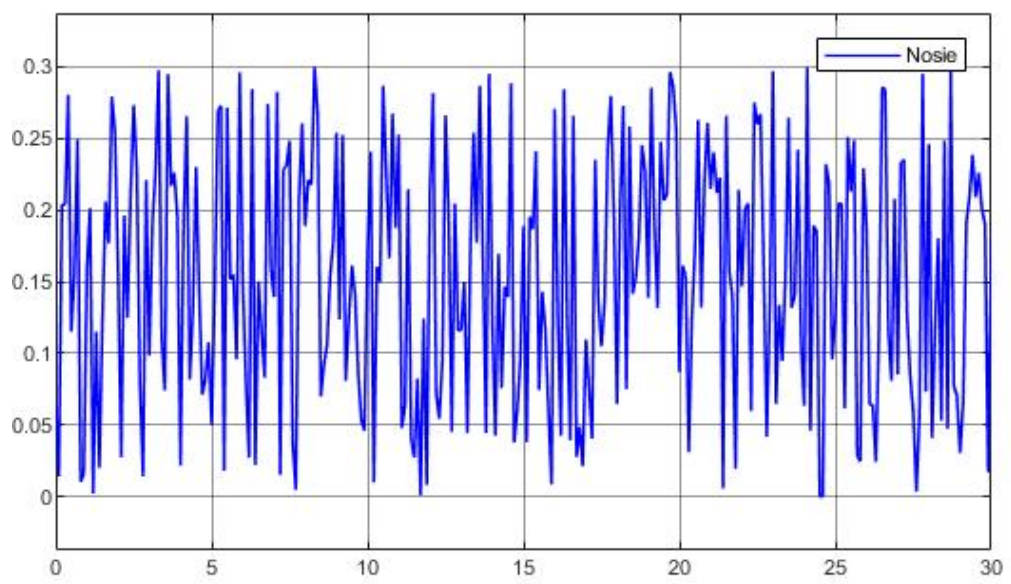


Remark 9: $\Phi$ and $d$ are randomly changed within their boundaries. In this system, based on the U-controller output of perfectly matched plant which is shown in Figure $8(d)$, it is clear that when controller output is small, system noise/disturbance will greatly affect system stability and control performance. Same as the boundary coefficient design for system (53), to avoid chattering, $\xi$ should be large enough. Let $\xi=0.1$, then the switching function is shown:

$$
\operatorname{sat}(\sigma)=\left\{\begin{array}{cc}
\operatorname{sgn}(\sigma), & |\sigma| \geq 0.1 \\
10 \sigma, & |\sigma|<0.1
\end{array}\right.
$$

Figure 15 shows the design framework of $\mathrm{U}_{\mathrm{SM}}$-control system for plant with internal uncertain parameter and system noise/disturbance. Figure 16 shows the comparison results of this practical simplified helicopter model with uncertainty and system noise/disturbance controlled by $\mathrm{U}$-control method and $\mathrm{U}_{\mathrm{SM}}$-control method. Figure 17 shows the compensated inverter output.

Figure $15 \mathrm{U}_{\mathrm{SM}^{-}}$-control of helicopter with system uncertainty and noise/disturbance

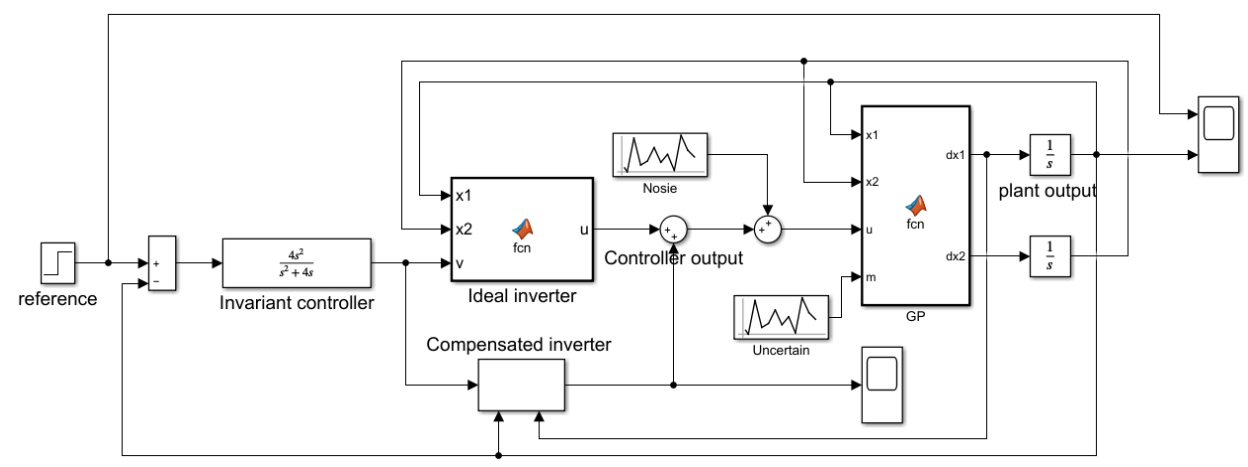

\subsection{Discussions}

For controlling perfectly matched plant, from Figures 8(a) and 8(b), both methods can make the controlled system be convergent and track the desired output reference. Additionally, from Figures 8(c) and 8(d), these two systems' controller outputs are also the same, that is, the compensated inverter output is none, which can be seen and proved by Figure 9. This means that the compensated inverter only works for system inversion disturbance. If the controlled plant is accurate and the controlled system has no interference, $\mathrm{U}_{\mathrm{SM}^{-}}$-control method will have the same controlled ability and performance with the U-control method.

For controlling mismatched plant, from Figures 12(a) and 12(b), $\mathrm{U}_{\mathrm{SM}}$-control method shows better performance than U-control method in tracking ability. From Figure 8(d) and Figure 12(d), U-controller output for perfectly matched plant and mismatched plant are almost the same, but the output performance is degraded due to the uncertainty. 
Figure 16 Comparison of mismatched simplified helicopter model with system noise/disturbance. (a) $\mathrm{U}_{\mathrm{SM}^{-}}$-control-based plant output and reference (b) U-control-based plant output and reference (c) $\mathrm{U}_{\mathrm{SM}^{-}}$-controller output (d) U-controller output

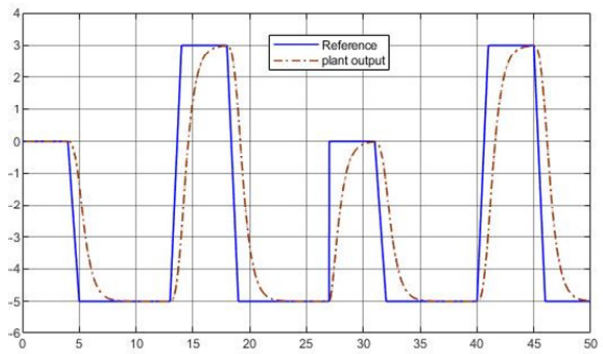

(a)

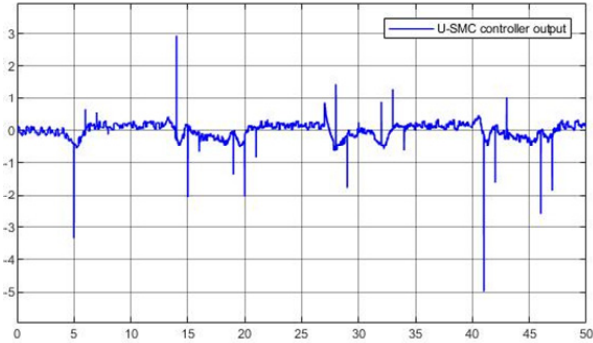

(c)

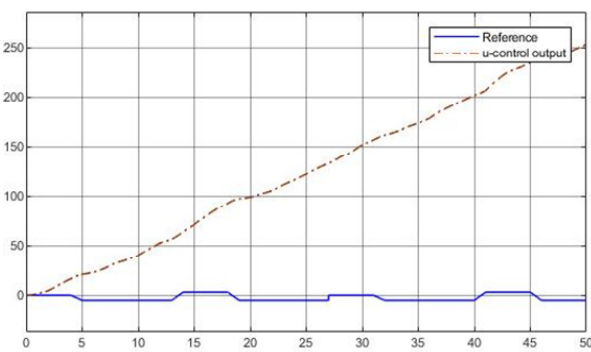

(b)

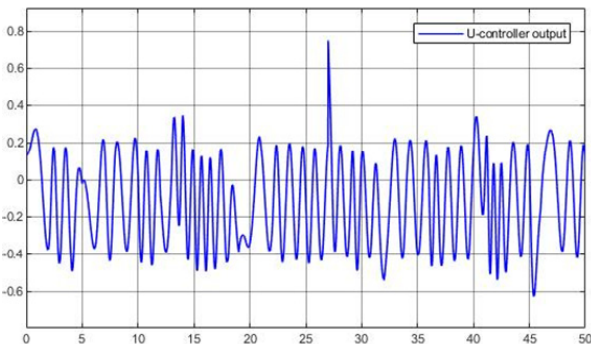

(d)

Figure $17 \mathrm{U}_{\mathrm{SM}^{-}}$-control-based compensated inverter output

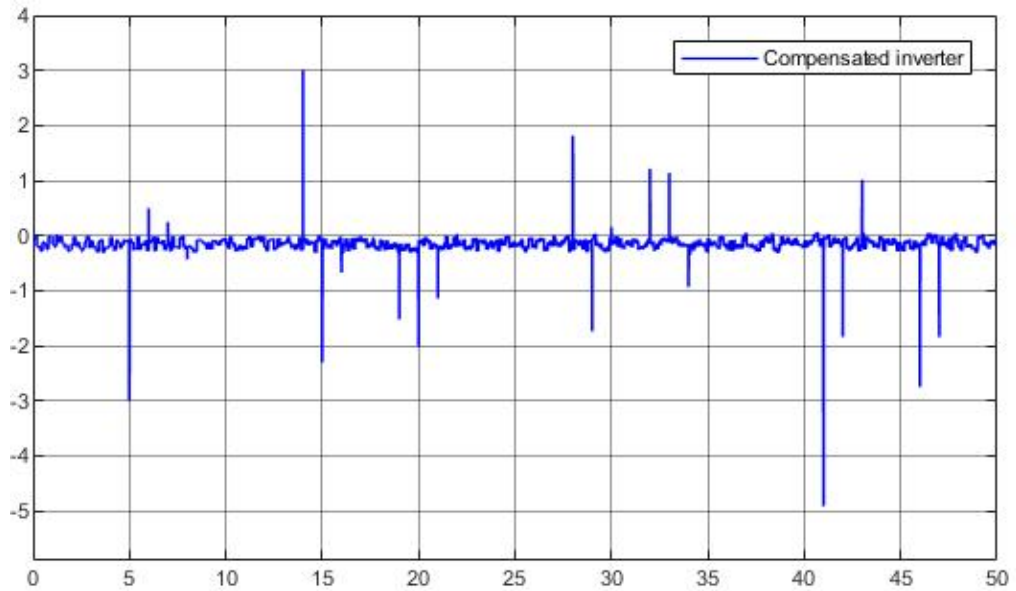

For controlling mismatched plant with system noise/disturbance, from Figures 16(a) and $16(\mathrm{~b})$, there is no doubt that $\mathrm{U}_{\mathrm{SM}^{-}}$-control-based control system shows better performance than U-control-based control system. With the influence of the uncertain system coefficient and noise, $\mathrm{U}_{\mathrm{SM}^{-}}$-control system can converge and track the reference perfectly, however, U-control system even cannot converge. From Figures 16(c) and 16(d), because of the different convergence abilities of these two systems, U-controller output is also divergent. The reason for the chattering in $U_{\mathrm{SM}}$-controller output is to counter the impact 
of high frequency noise interference. From Figures 9, 13 and 17, different from the compensated inverter output of perfectly matched simplified helicopter model, $\mathrm{U}_{\mathrm{SM}^{-}}$-control-based compensated inverter in mismatched controlled system starts working to force the dynamic inversion match the mismatched simplified helicopter model, that is, to reach $\left(G_{P}^{-1}+G_{e}^{-1}\right) G_{M}=1$ anyway.

\section{Conclusions}

This paper introduces a new sliding mode augmented $U$-control method ( $\mathrm{U}_{\mathrm{SM}}$-control) for tracking, which shows strong stability in control of uncertain and disturbing plants. The overall scheme is based on the U-control structure wherein its dynamic inverter is compensated by robust Sliding Mode Control (SMC). This work is able to combine the strong robustness of the SMC method and the control-oriented nature of the U-control to provide a comprehensive uncertain system control scheme; therefore, it is expected to prove clearly useful for practical control applications.

For the future work, firstly, the proposed method should be further tested and analysed to show its comparative advantages and disadvantages. The $\mathrm{U}_{\mathrm{SM}}$-control method is only SISO systems, therefore, this proposed scheme should be expanded to MultipleInput and Multiple-Output (MIMO) systems, especially underactuated and over-actuated systems.

\section{References}

Chalanga, A., Kamal, S., Fridman, L., Bandyopadhyay, B. and Moreno, J. (2016) 'Implementation of super-twisting control: super-twisting and higher order sliding-mode observer-based approaches', IEEE Transactions on Industrial Electronics, Vol. 63, No. 6, pp.3677-3685.

Ding, S., Zheng, W., Sun, J. and Wang, J. (2017) 'Second-order sliding-mode controller design and its implementation for buck converters', IEEE Transactions on Industrial Informatics, Vol. 14, No. 5, pp.1990-2000.

Edwards, C. and Spurgeon, S. (1998) Sliding Mode Control, Taylor \& Francis, London.

Geng, X., Zhu, Q., Liu, T. and Na, J. (2019) 'U-model based predictive control for nonlinear processes with input delay', Journal of Process Control, Vol. 75, pp.156-170.

Hussain, N., Ali, S., Ovinis, M., Arshad, M. and Al-Saggaf, U. (2020) 'Underactuated coupled nonlinear adaptive control synthesis using U-model for multivariable unmanned marine robotics', IEEE Access, Vol. 8, pp.1851-1865.

Isidori, A. (2013) Nonlinear Control Systems, Springer Science \& Business Media.

Li, H., Shi, P., Yao, D. and Wu, L. (2016) 'Observer-based adaptive sliding mode control for nonlinear Markovian jump systems’, Automatica, Vol. 64, pp.133-142.

Li, R., Zhu, Q., Kiely, J. and Zhang, W. (2020) 'Algorithms for U-model-based dynamic inversion (UM-dynamic inversion) for continuous time control systems', Complexity, pp.1-14.

Oberkampf, W., Helton, J., Joslyn, C., Wojtkiewicz, S. and Ferson, S. (2004) 'Challenge problems: uncertainty in system response given uncertain parameters', Reliability Engineering and System Safety, Vol. 85, Nos.1/3, pp.11-19.

Razavi, H., Merat, K., Salarieh, H., Alasty, A. and Meghdari, A. (2016) 'Observer based minimum variance control of uncertain piecewise affine systems subject to additive noise', Nonlinear Analysis: Hybrid Systems, Vol. 19, pp.153-167. 
Samadi, B. and Rodrigues, L. (2009) 'Stability of sampled-data piecewise affine systems: a timedelay approach', Automatica, Vol. 45, No. 9, pp.1995-2001.

Shtessel, Y. (2014) Sliding Mode Control and Observation, Springer, New York, pp.1-42.

Wang, Y., Shen, H., Karimi, H. and Duan, D. (2017) 'Dissipativity-based fuzzy integral sliding mode control of continuous-time TS fuzzy systems', IEEE Transactions on Fuzzy Systems, Vol. 26, No. 3, pp.1164-1176.

Zhu, Q.M. (1989) Identification and control of nonlinear systems (PhD thesis), University of Warwick, Coventry, UK.

Zhu, Q.M. and Guo, L. (2002) 'A pole placement controller for non-linear dynamic plants', Proceedings of the Institution of Mechanical Engineers, Part I: Journal of Systems and Control Engineering, Vol. 216, No. 6, pp.467-476.

Zhu, Q.M., Liu, L., Zhang, W. and Li, S. (2018) 'Control of complex nonlinear dynamic rational systems', Complexity, pp.1-12.

Zhu, Q.M., Wang, Y.J., Zhao, D.Y., Li, S.Y. and Billings, S. (2015) 'Review of rational (total) nonlinear dynamic system modelling, identification, and control', International Journal of Systems Science, Vol. 46, No. 12, pp.2122-2133.

Zhu, Q.M., Warwick, K. and Douce, J. (1991) 'Adaptive general predictive controller for nonlinear systems', IEEE Proceedings D Control Theory and Applications, Vol. 138, No. 1, pp.33-40.

Zhu, Q.M., Zhang, W., Na, J. and Sun, B. (2019) 'U-model based control design framework for continuous-time systems', Proceedings of the Chinese Control Conference (CCC), pp.106-111.

Zhu, Q.M., Zhang, W., Zhang, J. and Sun, B. (2019) 'U-neural network-enhanced control of nonlinear dynamic systems', Neurocomputing, Vol. 352, pp.12-21.

Zhu, Q.M., Zhao, D. and Zhang, J. (2015) 'A general U-block model-based design procedure for nonlinear polynomial control systems', International Journal of Systems Science, Vol. 47, No. 14 , pp.3465-3475. 